\title{
Two-dimensional anisotropic non-Hermitian Lieb lattice
}

\author{
L. C. Xie, ${ }^{1}$ H. C. Wu, ${ }^{1}$ X. Z. Zhang $\odot,{ }^{2, *}$ L. Jin $\odot,{ }^{1, \dagger}$ and Z. Song $\oplus^{1}$ \\ ${ }^{1}$ School of Physics, Nankai University, Tianjin 300071, China \\ ${ }^{2}$ College of Physics and Materials Science, Tianjin Normal University, Tianjin 300387, China
}

(Received 12 May 2021; accepted 25 August 2021; published 3 September 2021)

\begin{abstract}
We study an anisotropic two-dimensional non-Hermitian Lieb lattice, where the staggered gain and loss present in the horizontal and vertical directions, respectively. The intracell nonreciprocal coupling generates magnetic flux enclosed in the unit cell of the Lieb lattice and creates nontrivial topology. The active and dissipative topological edge states are along the horizontal and vertical directions, respectively. The two-dimensional non-Hermitian Lieb lattice also supports a passive topological corner state. At appropriate magnetic flux, the non-Hermiticity can alter the corner state from one corner to the opposite corner as the non-Hermiticity increases. The gapless phase of the Lieb lattice is characterized by different configurations of exceptional points in the Brillouin zone. The topology of the anisotropic non-Hermitian Lieb lattices can be verified in many experimental platforms including the optical waveguide lattices, photonic crystals, and electronic circuits.
\end{abstract}

DOI: 10.1103/PhysRevB.104.125406

\section{INTRODUCTION}

Over the last two decades, the ubiquitous effect of dissipation has proven to induce astonishing non-Hermitian features, rather than just being an inescapable nuisance. Within this young field, the treasure hunt is sprouting into fascinating new directions ranging from the complex optical media [1], nonequilibrium open systems with gain and/or loss [2-9], to strongly correlated systems as a result of finite-lifetime quasiparticles [10-16]. Most recently, topological characterization and dynamic control of non-Hermitian models have been hot areas of research [17-49]. Among the most relevant features observed in non-Hermitian systems, the appearance of an interface significantly alters the entire spectrum, leading to the exponential localization of all eigenmodes at the interface, which goes beyond the expectations for Hermitian systems. This unique non-Hermitian effect is dubbed the non-Hermitian skin effect [50]. As a consequence, the conventional bulk-boundary correspondence breaks down. A correct description requires one to extend Bloch band theory into the generalized Brillouin zone (BZ) [50-52]. Inspired by these exciting advances, different generalized versions of the bulk-boundary correspondence based on redefining the bulk topological indices to incorporate the impact of the non-Hermitian skin effect have been proposed [53-69]. Furthermore, the non-Hermitian skin effect itself is also a topological effect manifested by the spectral winding on

\footnotetext{
*zhangxz@tjnu.edu.cn

†jinliang@nankai.edu.cn
}

Published by the American Physical Society under the terms of the Creative Commons Attribution 4.0 International license. Further distribution of this work must maintain attribution to the author(s) and the published article's title, journal citation, and DOI. the complex energy plane with a reference energy. Importantly, the non-Hermitian topological phenomena have been observed experimentally in various experimental platforms including the optical waveguide lattices, photonic crystals, and electronic circuits [70-83].

The exceptional point (EP) uniquely presents in the non-Hermitian Hamiltonian [84-87]. At the EPs, eigenstate coalescence occurs. The order of the EP depends on the geometric multiplicity of the corresponding eigenvalue. In general, the band touching induces EPs in one-dimensional systems. Intriguingly, the EPs are topologically stable at generic points in the BZ in the sense that they will not disappear suddenly, but move, split, and merge in the BZ until merging in pairs.

In this paper, we study an anisotropic two-dimensional (2D) non-Hermitian Lieb lattice. The gain and loss present in the horizontal and vertical directions, respectively, and induce the active and dissipative topological edge states. The Lieb lattice supports the passive topological corner state, which is created at the appropriate cooperation between the nonHermiticity and the magnetic flux. The non-Hermitian term affects the corner states in a subtle way: when the intracell nonreciprocal coupling induced magnetic flux is $\pi / 2$, the corner states are always localized at one corner; however, when the intracell nonreciprocal coupling induced magnetic flux is $-\pi / 2$, the corner states slowly evolve into the opposite corner as the non-Hermiticity increases. The type and configuration of EPs in the BZ distinguish the gapless phases. Our findings shed light on the influence of non-Hermiticity for the 2D Lieb lattice.

The remainder of the paper is organized as follows. In Sec. II, we introduce the anisotropic 2D non-Hermitian Lieb lattice. Section III presents the topology of the nonHermitian Lieb lattice and the topological edge states in the gapped phase. The corner states under the influence of nonHermiticity are elaborated. Section IV highlights the enriched 


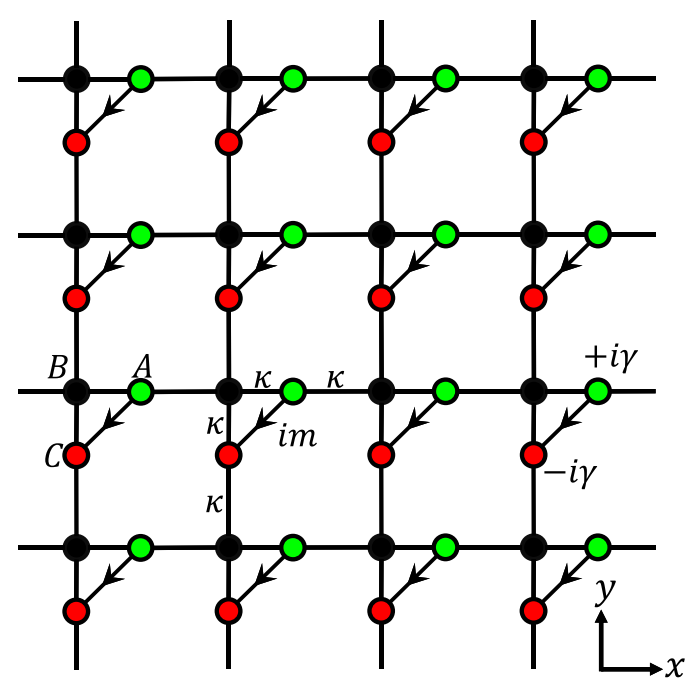

FIG. 1. Schematic illustration of the 2D non-Hermitian Lieb lattice. The system consists of three sublattices denoted by green, black, and red solid circles, respectively. The non-Hermiticity arises from the gain in red and loss in green. The black arrow indicates nonreciprocal coupling and each unit cell is threaded by a magnetic flux.

gapless band structures characterized by the EPs. Our conclusion is summarized in Sec. V.

\section{2D NON-HERMITIAN LIEB LATTICE}

We consider an anisotropic 2D non-Hermitian Lieb lattice. The Hamiltonian is written in the form of

$$
\begin{aligned}
H= & \sum_{l, n}\left(\kappa A_{l, n}^{\dagger} B_{l, n}+\kappa B_{l, n}^{\dagger} C_{l, n}+i m C_{l, n}^{\dagger} A_{l, n}\right. \\
& \left.+\kappa A_{l, n}^{\dagger} B_{l+1, n}+\kappa B_{l, n}^{\dagger} C_{l, n+1}\right)+ \text { H.c. } \\
& +i \gamma\left(A_{l, n}^{\dagger} A_{l, n}-C_{l, n}^{\dagger} C_{l, n}\right),
\end{aligned}
$$

where $A^{\dagger}, B^{\dagger}$, and $C^{\dagger}(A, B$, and $C$ ) denote the creation (annihilation) operators for the three sublattices in each unit cell. The non-Hermitian Lieb lattice is schematically illustrated in Fig. 1. $\kappa$ and $m$ are the coupling strengths. The intracell nonreciprocal coupling im between the sublattices $A$ and $C$ induces effective magnetic flux $\pi / 2$ enclosed in the unit cell. The nonHermiticity $\gamma$ originates from the balanced gain and loss that presented in the sublattices $A$ and $C$ in the horizontal and vertical directions, respectively. The proposed non-Hermitian Lieb lattice can be experimentally implemented in the platforms of ultracold atomic gas in optical lattices, photonic crystals, and coupled resonators based on the nowadays technology [88-94].

Applying the Fourier transformation $a_{l, n}=$ $N^{-1} \sum_{\mathbf{k}} e^{i \mathbf{k} \cdot \mathbf{r}} a_{\mathbf{k}} \quad(a=A, B, C)$ for the three sublattices, the Hamiltonian $H$ rewritten in the Nambu representation reads

$$
H=\sum_{\mathbf{k}} H(\mathbf{k})=\sum_{\mathbf{k}} \psi_{\mathbf{k}}^{\dagger} h(\mathbf{k}) \psi_{\mathbf{k}}
$$

where the basis is $\psi_{\mathbf{k}}=\left[A_{\mathbf{k}}, B_{\mathbf{k}}, C_{\mathbf{k}}\right]^{T}$ and $h(\mathbf{k})$ is a $3 \times 3$ matrix,

$$
h(\mathbf{k})=\left(\begin{array}{ccc}
i \gamma & \kappa\left(e^{i k_{x}}+1\right) & -i m \\
\kappa\left(e^{-i k_{x}}+1\right) & 0 & \kappa\left(e^{i k_{y}}+1\right) \\
i m & \kappa\left(e^{-i k_{y}}+1\right) & -i \gamma
\end{array}\right) .
$$

The Hamiltonians in the momentum subspaces commute with each other, $\left[H(\mathbf{k}), H\left(\mathbf{k}^{\prime}\right)\right]=0$.

At $m=\gamma=0$, the lattice is a standard Lieb lattice and supports a flat band. In the absence of the gain and loss $\gamma=0$, the lattice has a flat band and the band energy is tuned by the coupling strength $m$ [95-102]. The wave transport in the flat band is completely suppressed because of the momentum-independent dispersion relation, leading to a strong localization of the eigenstates. This provides an ideal platform to investigate various interesting strongly correlated phenomena [103-106].

The presence of gain and loss drastically alters the spectrum and results in exotic phenomena compared to the standard Lieb lattice. We investigate $h(\mathbf{k})$ to show the insights of the non-Hermitian Lieb lattice. The energy band properties of $h(\mathbf{k})$ are determined from solving the secular equation $\operatorname{det}[h(\mathbf{k})-E(\mathbf{k}) I]=0$, where $I$ is the identity matrix. The algebra after the basis transformation shows a cubic equation

$$
E^{3}(\mathbf{k})+p\left(h_{x}, h_{y}\right) E(\mathbf{k})+q\left(h_{x}, h_{y}\right)=0,
$$

where

$$
\begin{gathered}
p\left(h_{x}, h_{y}\right)=\gamma^{2}-h_{x}^{2}-h_{y}^{2}-m^{2}, \\
q\left(h_{x}, h_{y}\right)=i \gamma\left(h_{y}^{2}-h_{x}^{2}\right)+2 h_{x} h_{y} m \sin \left[\left(k_{x}+k_{y}\right) / 2\right]
\end{gathered}
$$

with $h_{\epsilon}^{2}=\left|\kappa\left(e^{i k_{\epsilon}}+1\right)\right|^{2}(\epsilon=x, y)$. The energy bands and eigenstates are straightforwardly obtained through solving the cubic equation.

\section{TOPOLOGICAL PHASES}

In this section, we discuss the topological phase of the system based on the non-Hermitian topological band theory [107]. For the non-Hermitian Hamiltonian, the separable energy band means that any two bands of the system are not degenerate at any point $\mathbf{k}$ in the momentum space [i.e., $E_{\alpha}(\mathbf{k}) \neq E_{\beta}(\mathbf{k}) ; \alpha, \beta$ are the band index]. The system experiences a topological phase transition when the closing of separable bands occurs.

For separable bands, the topology of the anisotropic 2D non-Hermitian Lieb lattice can be characterized by the Chern number. Clearly, there is a fixed magnetic flux in the unit cell because of the nonreciprocal coupling strength between the next-nearest neighbor, which breaks the time-reversal symmetry and ensures the existence of the Chern number. For nonzero Chern number, the topological edge states exist for the system under OBC in the $x$ or $y$ direction; for zero Chern number, the system has trivial edge states or no edge states when imposing OBC in the $x$ or $y$ direction.

Separable bands and Chern number. Figures 2 and 3 are the phase diagrams. The nonzero Chern number is marked in Fig. 3 . The white regions except for the topologically nontrivial region marked with $C= \pm 1$ are all topologically trivial phases 


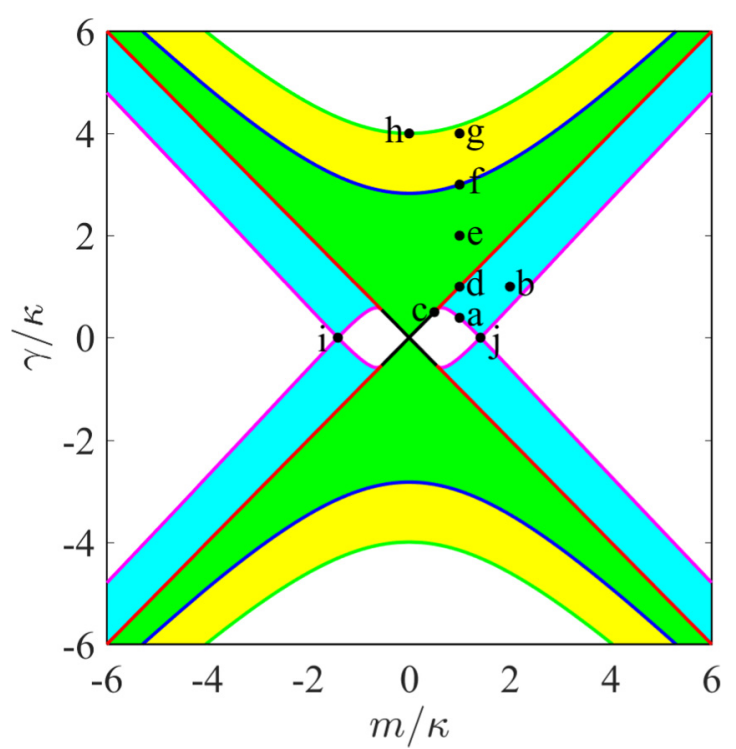

FIG. 2. Phase diagram in the $m-\gamma$ parameter space. The color regions show the three gapless phases distinguished by the EPs in the BZ. The white regions show the gapped phases distinguished by the Chern numbers.

with the zero Chern number. The non-Hermiticity greatly affects the topological property of the Lieb lattice. When the non-Hermitian term is introduced, the gapped regions shrink and the gapless regions appear; a typical feature is the existence of the EPs. Moreover, the large non-Hermiticity destroys

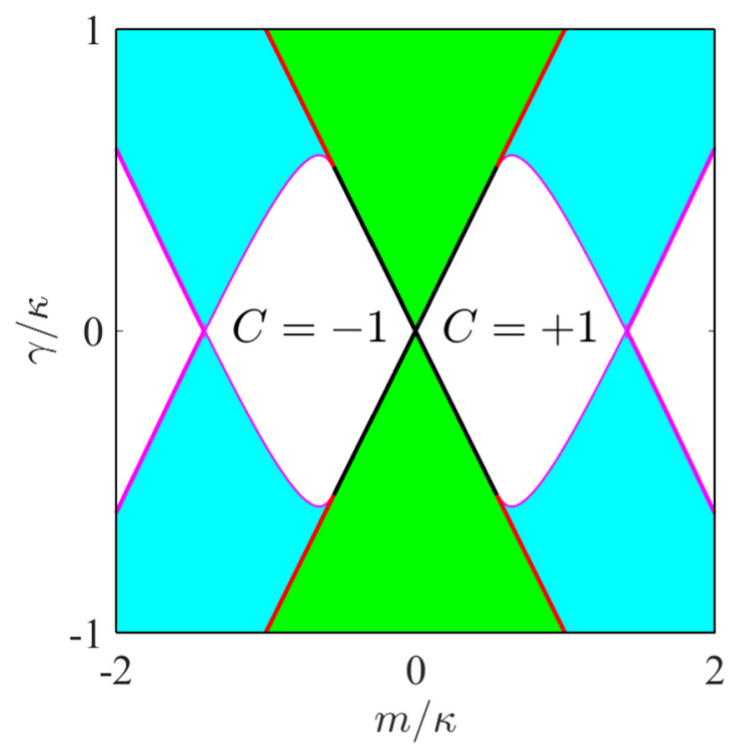

FIG. 3. Phase diagram in the $m-\gamma$ parameter space focused on the gapped regions. Here $\kappa$ is taken as the unit without loss of generality. In gapped regions, the system can be either in topologically trivial or nontrivial phase characterized by the Chern number. $C= \pm 1$ indicates the Chern number of the lowest band of the topologically nontrivial phase. Except for the white region marked with $C=1$, all other white regions satisfy $C=0$. For the topologically nontrivial phase, edge states exist for the system under OBC in the $x$ or $y$ direction.
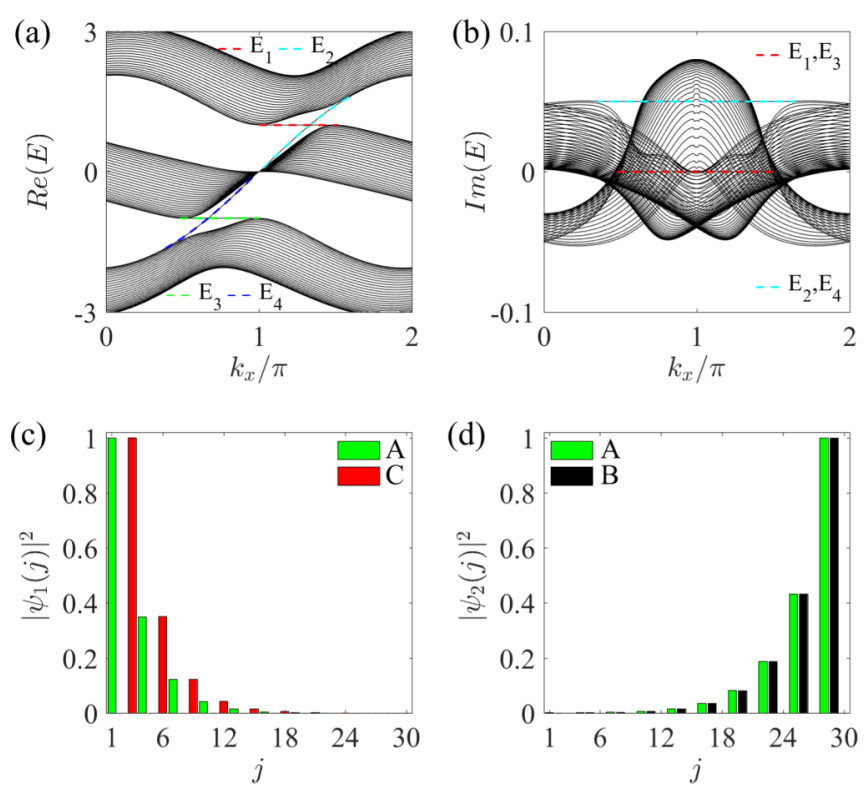

FIG. 4. Energy spectrum of the topologically nontrivial phase under $\mathrm{PBC}$ in the $x$ direction, but under $\mathrm{OBC}$ in the $y$ direction. Here $\kappa$ is taken as the unit without loss of generality, and the total number of the unit cells $N$ is 10. (a), (b) $m=-1, \gamma=0.1$ depict the topologically nontrivial phase with gapless edge states. (c), (d) The probability distribution of $\left|\psi_{1}\right\rangle$ and $\left|\psi_{2}\right\rangle$ by supposing $k_{x}=1.4 \pi$. Different color bars indicate different sublattices, respectively.

the nontrivial topology of the Lieb lattice as shown in the phase diagram Fig. 2.

The Chern number of the separable energy band is well defined as the integral of Berry curvature on the entire Brillouin zone,

$$
C=\frac{1}{2 \pi} \iint_{\mathrm{BZ}} d k_{x} d k_{y} \Omega,
$$

where $\Omega=\nabla \times \mathcal{A}$ with $\mathcal{A}=-i\langle\varphi(\mathbf{k})|\nabla| \varphi(\mathbf{k})\rangle$ and $|\varphi(\mathbf{k})\rangle$ is the eigenstate of the band. The Chern number evaluated under $\mathrm{PBC}$ predicts the number of gapless edge states of the system under OBC [107]. In the topologically nontrivial phase, the Chern number for the lower band is $C= \pm 1$, the Chern number for the middle band is $C=0$, and the Chern number for the upper band is $C=\mp 1$. In the topologically trivial phase, the Chern numbers for the energy bands are all zeros.

As a consequence, the gapless edge states between the middle band and the other bands exist for the Lieb lattice under OBC in the $x$ or the $y$ direction. Figure 4(a) depicts the energy bands when the system is under PBC in the $x$ direction, but under $\mathrm{OBC}$ in the $y$ direction. Figure 5(a) shows the energy band when PBC is applied in the $y$ direction but $\mathrm{OBC}$ is applied in the $x$ direction. The non-Hermitian Hamiltonian $h(\mathbf{k})$ in the momentum space has the particle-hole symmetry, $\mathcal{T}_{-} h^{*}(\mathbf{k}) \mathcal{T}_{-}^{-1}=-h(-\mathbf{k})$; the unitary operator $\mathcal{T}_{-}$is defined as $\mathcal{T}_{-}=\operatorname{diag}(1,-1,1)$. Thus, the band energies satisfy $E_{\mathbf{k}}=$ $-E_{-\mathbf{k}}^{*}$ as reflected from Fig. 4(a).

Edge states. In the topologically nontrivial phase of the non-Hermitian Lieb lattice, we examine the properties of the gapless edge states. The edge states localized on the boundary in the $x$ and $y$ direction exhibit the gain and loss, respectively. 

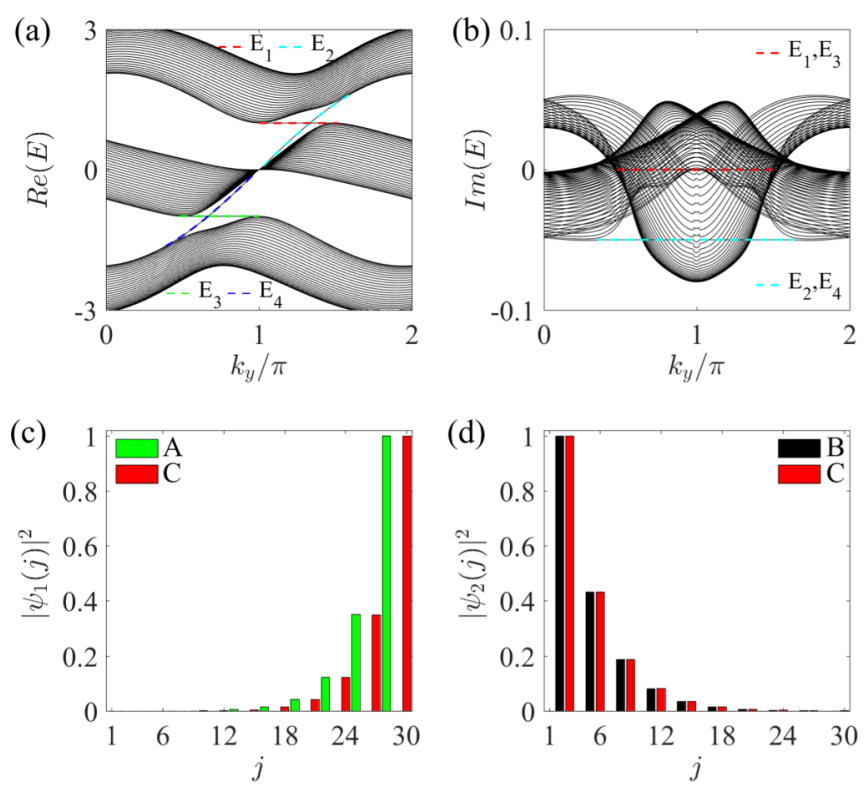

FIG. 5. Energy spectrum of the gapless edge states under PBC in the $y$ direction, but under $\mathrm{OBC}$ in the $x$ direction. The (a) real and (b) imaginary parts of energy band at $m=-1, \gamma=0.1$. The edge states (c) $\left|\psi_{1}\right\rangle$ and (d) $\left|\psi_{2}\right\rangle$ at $k_{y}=1.4 \pi$. Color sticks indicate the probabilities for the corresponding sublattices. The total number of unit cells $N$ is 10 and $\kappa=1$.

We discuss the $C=-1$ region as an example. The energy spectra for the lattice under PBC in the $x$ direction and OBC in the $y$ direction are shown in Figs. 4(a) and 4(b). Four edge states appear in pairs within the band gap. Straightforward algebra shows that

$$
\begin{gathered}
E_{1}=\sqrt{m^{2}-\gamma^{2}}, \\
E_{2}=\sqrt{4 \kappa^{2} \cos ^{2}\left(k_{x} / 2\right)-\gamma^{2} / 4}+i \gamma / 2, \\
E_{3}=-\sqrt{m^{2}-\gamma^{2}}, \\
E_{4}=-\sqrt{4 \kappa^{2} \cos ^{2}\left(k_{x} / 2\right)-\gamma^{2} / 4}+i \gamma / 2 .
\end{gathered}
$$

The edge state energies between the middle and lowest bands satisfy $E_{3}=-E_{1}$ and $E_{4}=-E_{2}^{*}$ under the particle-hole symmetry. $E_{1}$ and $E_{3}$ are opposite in pair and independent of the momentum $k_{x}$. $E_{2}$ and $E_{4}$ are complex with constant gain rate $i \gamma / 2$. The wave function of the edge state for $E_{\mu}$ is denoted as $\left|\psi_{\mu}\right\rangle$ with $\mu=1,2,3,4$. We set the expression of the edge states as $\left|\psi_{\mu}\right\rangle=\left(\psi_{1 A}, \psi_{1 B}, \psi_{1 C}, \ldots, \psi_{N A}, \psi_{N B}, \psi_{N C}\right)$ with $N$ being the total number of the unit cells. To analytically obtain the wave function of the edge states, we consider the lattice size at the limitation of infinity large $N \rightarrow \infty$.

For the edge state $\left|\psi_{1}\right\rangle$, the component of $\left|\psi_{1}\right\rangle$ at the sublattice $B$ is $\psi_{n B}=0$ with $n$ being the index of the unit cell in the $y$ direction. The components of $\left|\psi_{1}\right\rangle$ in the first unit cell at the bottom are $\left(\psi_{1 A}, \psi_{1 B}, \psi_{1 C}\right)=\left(1,0, e^{i \phi}\right)$ with $\phi=\operatorname{sgn}(m) \arccos (\gamma / m)$. The components of $\left|\psi_{1}\right\rangle$ in the $n$th unit cell satisfy the recursion relation $\left(\psi_{n A}, \psi_{n B}, \psi_{n C}\right)=$ $\rho^{n-1}\left(\psi_{1 A}, \psi_{1 B}, \psi_{1 C}\right)$ with $\rho=-e^{-i \phi}-e^{-i\left(\phi+k_{x}\right)}-1$. The probabilities of edge states for the sublattices $A$ and $C$ in every unit cell are identical and decay exponentially from bottom to top of the Lieb lattice as shown in Fig. 4(c). For the edges state $\left|\psi_{3}\right\rangle$, we have a similar wave function distribution. The components of $\left|\psi_{3}\right\rangle$ in the first unit cell at the bottom are $\left(\psi_{1 A}, \psi_{1 B}, \psi_{1 C}\right)=\left(1,0, e^{-i \phi}\right)$ and decay as the index of the unit cells at the rate $\rho=-e^{i \phi}-e^{i\left(\phi-k_{x}\right)}-1$.

For the edge state $\left|\psi_{2}\right\rangle$, the component of $\left|\psi_{2}\right\rangle$ at the sublattice $C$ vanishes, $\psi_{n C}=0$. The components of $\left|\psi_{2}\right\rangle$ in the first unit cell at the top are $\left(\psi_{N A}, \psi_{N B}, \psi_{N C}\right)=$ $\left(e^{i\left(\phi+k_{x} / 2\right)}, 1,0\right)$ with $\phi=\arcsin \left\{\gamma /\left[4 \cos \left(k_{x} / 2\right)\right]\right\}$ for $m>$ 0 , and $\pi-\arcsin \left\{\gamma /\left[4 \cos \left(k_{x} / 2\right)\right]\right\}$ for $m<0$. The components of $\left|\psi_{2}\right\rangle$ in the $n$th unit cell satisfy the recursion relation $\left(\psi_{n A}, \psi_{n B}, \psi_{n C}\right)=\rho^{N-n}\left(\psi_{N A}, \psi_{N B}, \psi_{N C}\right)$ with $\rho=$ $-1-i m e^{i\left(\phi+k_{x} / 2\right)}$. The probabilities of the edge states for the sublattices $A$ and $B$ in every unit cell are identical and decay exponentially from top to bottom of the Lieb lattice as shown in Fig. 4(d). For the edge state $\left|\psi_{4}\right\rangle$, the result is similar. The components of $\left|\psi_{4}\right\rangle$ in the first unit cell at the top are $\left(\psi_{N A}, \psi_{N B}, \psi_{N C}\right)=\left(-e^{i\left(k_{x} / 2-\phi\right)}, 1,0\right)$ and decay as the index of the unit cells at the rate $\rho=-1+$ $i m e^{i\left(k_{x} / 2-\phi\right)}$ with $\phi=\arcsin \left\{\gamma /\left[4 \cos \left(k_{x} / 2\right)\right]\right\}$ for $m<0$, and $\pi-\arcsin \left\{\gamma /\left[4 \cos \left(k_{x} / 2\right)\right]\right\}$ for $m>0$.

The energy spectra for the lattice under PBC in the $y$ direction and OBC in the $x$ direction are shown in Figs. 5(a) and 5(b). There are still four edge states in pairs within the band gap, in the form of

$$
\begin{gathered}
E_{1}=\sqrt{m^{2}-\gamma^{2}}, \\
E_{2}=\sqrt{4 \kappa^{2} \cos ^{2}\left(k_{y} / 2\right)-\gamma^{2} / 4}-i \gamma / 2, \\
E_{3}=-\sqrt{m^{2}-\gamma^{2}}, \\
E_{4}=-\sqrt{4 \kappa^{2} \cos ^{2}\left(k_{y} / 2\right)-\gamma^{2} / 4}-i \gamma / 2 .
\end{gathered}
$$

$E_{1}$ and $E_{3}$ are independent of the momentum $k_{y}$ with $E_{3}=$ $-E_{1} . E_{2}$ and $E_{4}$ are complex with constant loss rate $-i \gamma / 2$ with $E_{4}=-E_{2}^{*}$. We analyze the wave functions of the four edge states in the same way as above.

For the edge state $\left|\psi_{1}\right\rangle$, the component of $\left|\psi_{1}\right\rangle$ at the sublattice $B$ is $\psi_{v B}=0$ with $v$ being the index of the unit cell in the $x$ direction. The components of $\left|\psi_{1}\right\rangle$ in the first unit cell at the right are $\left(\psi_{N A}, \psi_{N B}, \psi_{N C}\right)=$ $\left(e^{-i \phi}, 0,1\right)$ with $\phi=\operatorname{sgn}(m) \arccos (\gamma / m)$. The components of $\left|\psi_{1}\right\rangle$ in the $v$ th unit cell satisfy the recursion relation $\left(\psi_{v A}, \psi_{v B}, \psi_{v C}\right)=\rho^{N-v}\left(\psi_{N A}, \psi_{N B}, \psi_{N C}\right)$ with $\rho=$ $-1-e^{i \phi}-e^{i\left(k_{y}+\phi\right)}$. The probabilities of edge states for the sublattices $A$ and $C$ in every unit cell are identical and decay exponentially from right to left of the Lieb lattice as shown in Fig. 5(c). For the edges state $\left|\psi_{3}\right\rangle$, we have a similar wave function distribution. The components of $\left|\psi_{3}\right\rangle$ in the first unit cell at the right are $\left(\psi_{1 A}, \psi_{1 B}, \psi_{1 C}\right)=\left(e^{i \phi}, 0,1\right)$ and decay as the index of the unit cells at the rate $\rho=-1-e^{-i \phi}-e^{i\left(k_{y}-\phi\right)}$.

For the edge state $\left|\psi_{2}\right\rangle$, the component of $\left|\psi_{2}\right\rangle$ at the sublattice $A$ vanishes, $\psi_{v A}=0$. The components of $\left|\psi_{2}\right\rangle$ in the first unit cell at the left are $\left(\psi_{1 A}, \psi_{1 B}, \psi_{1 C}\right)=$ $\left(0,1, e^{-i\left(\phi+k_{y} / 2\right)}\right)$ with $\phi=\arcsin \left\{\gamma /\left[4 \cos \left(k_{y} / 2\right)\right]\right\}$ for $m>$ 0 , and $\pi-\arcsin \left\{\gamma /\left[4 \cos \left(k_{y} / 2\right)\right]\right\}$ for $m<0$. The components of $\left|\psi_{2}\right\rangle$ in the $v$ th unit cell satisfy the recursion relation $\left(\psi_{v A}, \psi_{v B}, \psi_{v C}\right)=\rho^{v-1}\left(\psi_{1 A}, \psi_{1 B}, \psi_{1 C}\right)$ with $\rho=$ $-1+i m e^{-i\left(\phi+k_{y} / 2\right)}$. The probabilities of the edge states for 

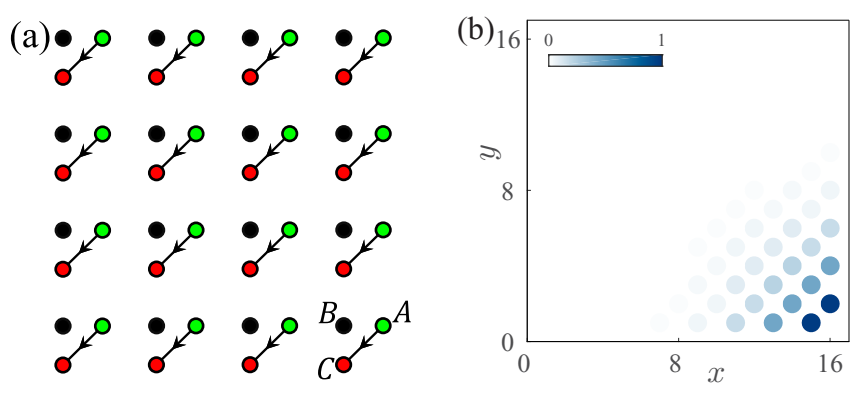

FIG. 6. Corner states of the Lieb lattice at the energies $E_{1}$ and $E_{3}$. (a) The dimerized structure of the corner state, being paritytime-symmetric. (b) The probability distribution of the corner state. The parameters are $\kappa=1, m=-1, \gamma=0.1$, and the lattice consists of $8 \times 8$ unit cells. The color bar indicates the probability of wave function and the maximum is renormalized to 1 .

the sublattices $B$ and $C$ in every unit cell are identical and decay exponentially from left to right of the Lieb lattice as shown in Fig. 5(d). For the edge state $\left|\psi_{4}\right\rangle$, the result is similar. The components of $\left|\psi_{4}\right\rangle$ in the first unit cell at the right are $\left(\psi_{N A}, \psi_{N B}, \psi_{N C}\right)=\left(0,1,-e^{i\left(\phi-k_{y} / 2\right)}\right)$ and decay as the index of the unit cells at the rate $\rho=-1-$ $i m e^{i\left(\phi-k_{y} / 2\right)}$ with $\phi=\arcsin \left\{\gamma /\left[4 \cos \left(k_{y} / 2\right)\right]\right\}$ for $m<0$, and $\pi-\arcsin \left\{\gamma /\left[4 \cos \left(k_{y} / 2\right)\right]\right\}$ for $m>0$.

From Figs. 4(c) and 5(c), the edge state energies are independent of the momentum and the edge states locate on the sublattices $A$ and $C$ for the non-Hermitian Lieb lattice under the open boundary in either the $x$ or the $y$ direction. If the boundaries of the non-Hermitian Lieb lattice on both the $x$ and the $y$ directions are open, the corner state appears at the bottom right of the non-Hermitian Lieb lattice. The structure of the corner state is schematically illustrated in Fig. 6(a) and the probability distribution of the corner state is shown in Fig. 6(b). The destructive interference plays the crucial role to form the dimerized pattern of the corner state.
In the Hermitian case with zero $\gamma$, numerical simulation for the finite system shows that there exist two corner states with energy $E_{c}= \pm|m|$. The corresponding corner states appear at the bottom right of the lattice as shown in Figs. 7(b) and $7(\mathrm{~g})$, where the sublattice $B$ is unoccupied. For the case with nonzero $\gamma$, the non-Hermitian term affects the corner states in a subtle way. Numerical results are plotted in Fig. 7; the influence of the gain and loss is demonstrated.

We find that (i) for $\gamma / m<0$, the magnetic flux enclosed in the unit cell is $\pi / 2$. The corner states always appear at the bottom right of the lattice, and become more localized with the increase of non-Hermiticity $|\gamma|$. while (ii) for $\gamma / m>0$, the magnetic flux enclosed in the unit cell is $-\pi / 2$. The corner state becomes more extended as $|\gamma|$ increases, and the bottom-right corner states slowly evolve into the top-left corner state. A new corner state appears at the top left of the lattice as $|\gamma|$ across about 0.5 . Interestingly, the occupation of the $B$ sublattice is dominant in this case.

\section{BAND STRUCTURE OF GAPLESS PHASE CHARACTERIZED BY THE EPS}

In this section, we investigate the band structure of the non-Hermitian Lieb lattice, where the existence of the EP in the spectrum is featured for the gapless phase. The EP is unique for the non-Hermitian physics and is associated with the level coalescence, where not only the eigenenergies but also the eigenstates become the same. Many interesting effects without Hermitian counterparts arise around the EP, ranging from the square root frequency dependence [108], the nontrivial topological property resulting from the Riemann sheet structures $[109,110]$, to unidirectional reflectionless and coherent perfect absorption $[83,111,112]$.
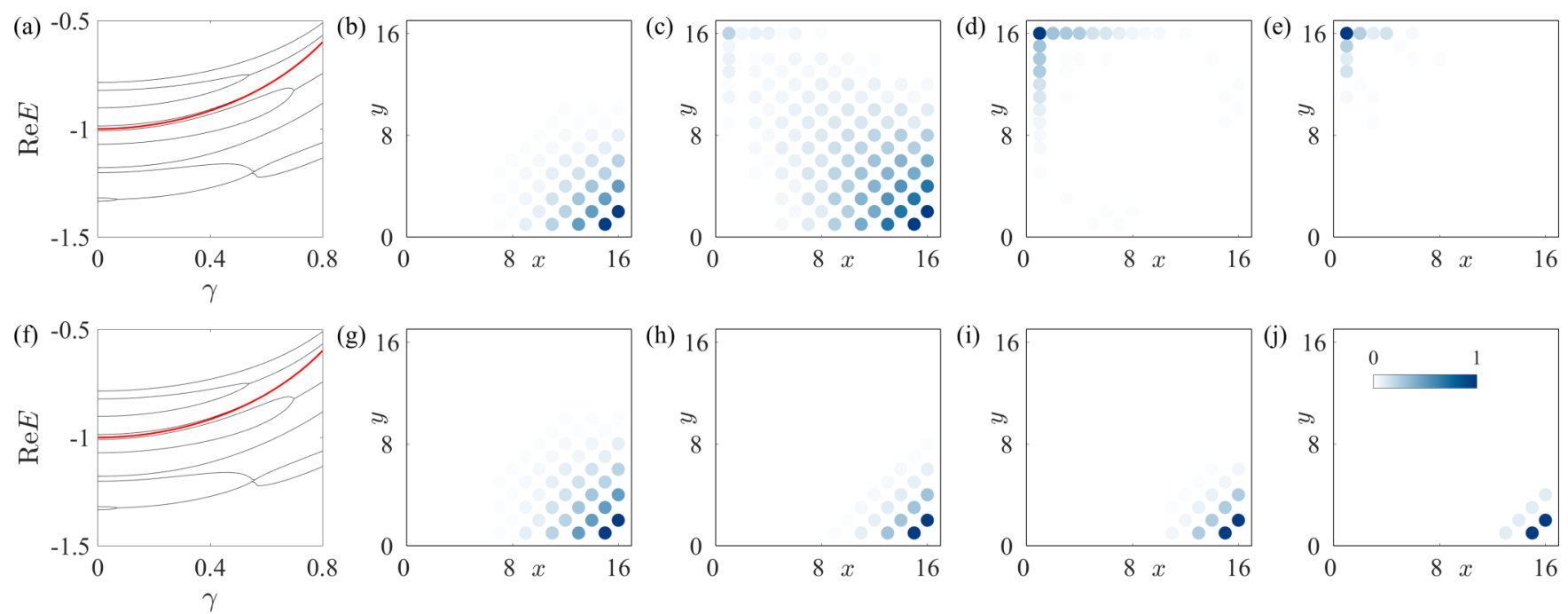

FIG. 7. The profiles of corner state in the real space for finite-size non-Hermitian Lieb lattice affected by the non-Hermiticity. The $2 \mathrm{D}$ lattice consists of $8 \times 8$ unit cells. (a) and (f) The plots of energy levels close to the corner states (red line) as functions of $\gamma$. $m=1$ for the upper panels and $m=-1$ for the lower panels. (b), (g) $\gamma=0$; (c), (h) $\gamma=0.4$; (d), (i) $\gamma=0.5$; (e), (j) $\gamma=0.8$. The other parameter is $\kappa=1$. The color bar indicates the probability of wave function and the maximum is renormalized to 1 . 

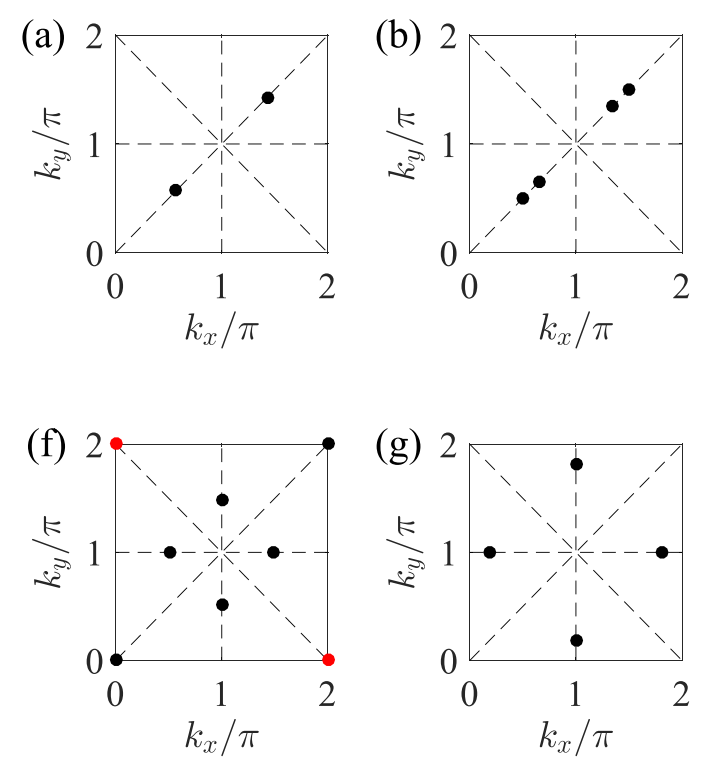
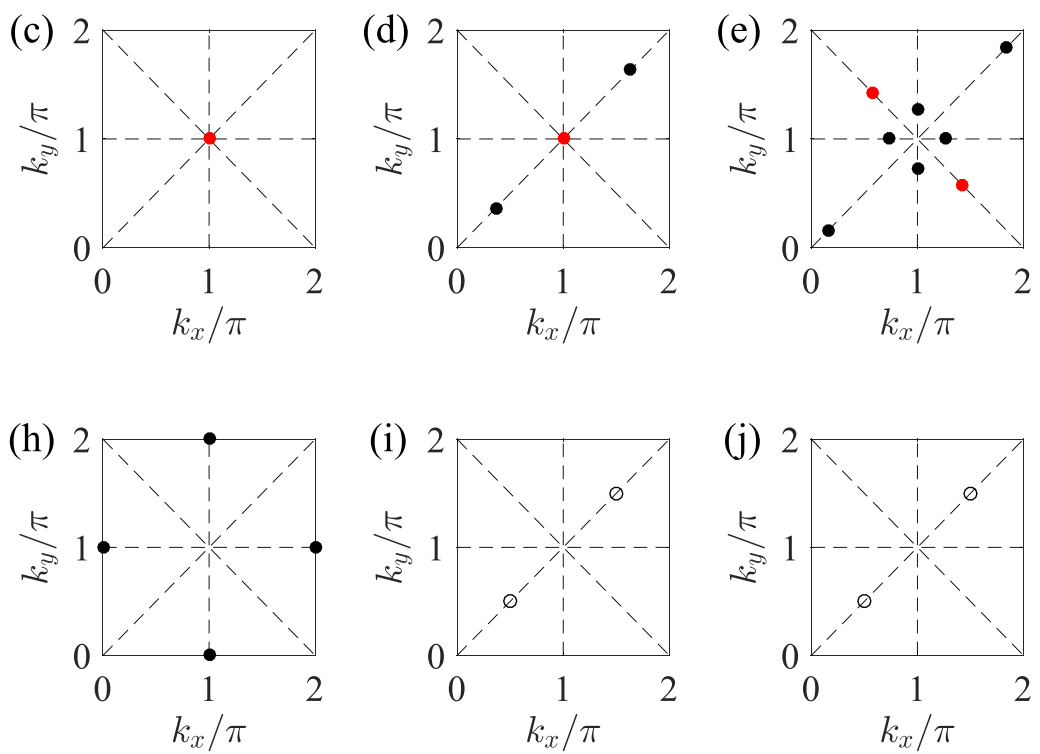

FIG. 8. Complex energy band structures of the non-Hermitian Bloch Hamiltonian $h(\mathbf{k})$. The system parameters are determined by the representative points and trace the variation of EP in different regions of Fig. 2. The EPs projected on the $k_{x}-k_{y}$ plane: (a) $m=1, \gamma=0.388$; (b) $m=1, \gamma=0.5$; (c) $m=0.5, \gamma=0.5$; (d) $m=1, \gamma=1$; (e) $m=1, \gamma=2$; (f) $m=1, \gamma=3$; (g) $m=1, \gamma=4$; (h) $m=1, \gamma=4.162$; (i) $m=-\sqrt{2}, \gamma=0$; and (j) $m=\sqrt{2}, \gamma=0$. The other system parameter is $\kappa=1$. The solid black dots, solid red dots, and black circles represent EP2, EP3, and DP2, respectively. Notably, the variation of $\gamma$ can induce the splitting and the merging of the different types of EP so that the system can exhibit rich structure.

To calculate the EP of the non-Hermitian Lieb lattice, we define $\Delta$ as the discriminant of the cubic equation (4). The real and imaginary parts of $\Delta$ read

$$
\begin{gathered}
\operatorname{Im}(\Delta)=m \gamma h_{x} h_{y} \sin \left[\left(k_{x}+k_{y}\right) / 2\right]\left(h_{y}^{2}-h_{x}^{2}\right), \\
\operatorname{Re}(\Delta)=p^{3} / 27+\operatorname{Re}\left(q^{2}\right) / 4 .
\end{gathered}
$$

$\Delta=0$ with nonzero $\gamma$ signifies the EP in the spectrum, where the energy bands are contacted. The phase diagram for the band structure determined from Eqs. (16) and (17) is depicted in Fig. 2. The non-Hermitian system is gapless when the energy bands are inseparable. In the gapless phase, at least two of the bands touch and $\mathbf{k}_{\mathrm{EP}}$ is the EP in the BZ; besides, the EPs might present in different energy bands at the same momentum $\mathbf{k}_{\mathrm{EP}}$. The gapless phase is divided into three regions in terms of the types of EPs rather than the exceptional ring [113], denoted as yellow, green, and cyan. In the yellow regions, there are four EP2s (two-state coalescence); six EP2s and two EP3s (three-state coalescence) present in the green regions; and the cyan regions have four EP2s. The EP merge or split when the system parameters cross the solid lines as the boundaries between colored regions.

We take $\kappa=1$ as an illustration and analytically determine the EPs and the boundaries within the gapless phases from $\operatorname{Im}(\Delta)=0$ and $\operatorname{Re}(\Delta)=0$. The EPs may appear at $k_{x}=\pi$, $k_{y}=\pi$, and $\left|k_{x}\right|=\left|k_{y}\right|$.

(i) The EP2s appear at $k_{x}=k_{y}$; we obtain $h_{x}^{2}=h_{y}^{2}$ and $\operatorname{Im}(\Delta)=0$. The cyan and green regions in the phase diagram have EP2s in this case, where the energies at the EP2s are real with $\operatorname{Im}(E)=0$. From $\operatorname{Re}(\Delta)=0$, we obtain

$$
4\left(\gamma^{2}-m^{2}-2 h_{x}^{2}\right)^{3}=27 h_{x}^{6} m^{2}\left(h_{x}^{2}-4\right),
$$

which determines the green and cyan regions in Fig. 2. The purple boundary and the boundary $|\gamma|=|m|$ in red enclose the cyan region with four EP2s, but the blue boundary and the boundary $|\gamma|=|m|$ enclose the green region with two EP2s. The representative points in the two regions are shown in Fig. 8(b) and Fig. 8(e), respectively. We notice that on the dashed line $k_{x}=k_{y}$, the former has four EP2s, and the latter has only two. At the boundary, the number of EP2s will be reduced due to merging. In Fig. 8(a) and Fig. 8(d), we notice that the four EP2s in the cyan region merge into two at the purple or red boundaries. In Fig. 8(c) and Fig. 8(f), we notice that the two EP2s in the green region merge into one EP at the blue or black boundaries.

(ii) The EP3s appear at $k_{x}=-k_{y}$; we obtain $\sin \left(k_{x}+\right.$ $\left.k_{y}\right) / 2=0$ and $\operatorname{Im}(\Delta)=0$. From $\operatorname{Re}(\Delta)=0$, we obtain

$$
\gamma^{2}-m^{2}=2 h_{x}^{2},
$$

which determines the green region. The boundary $\gamma^{2}-m^{2}=$ 8 in blue and the boundary $|\gamma|=|m|$ enclose the green region with two EP3s. This indicates that the green region has EP3s in addition to EP2s. The EP3s are the red dots in Fig. 8(e), where the three bands coalesce at zero energy $E=0$. Two EP3s merge to one at $\left(k_{x}, k_{y}\right)=(0,0)$ in Fig. 8(f) at the blue boundary and one at $\left(k_{x}, k_{y}\right)=(\pi, \pi)$ in Figs. 8(c) and 8(d) at $|\gamma|=|m|$. The non-Hermitian Lieb lattice does not hold the antiunitary symmetries and the EP3s are not topologically stable [114-116].

(iii) The EP2s appear at $k_{x}=\pi$ or $k_{y}=\pi$, which leads to $h_{x}=0$ or $h_{y}=0$ and $\operatorname{Im}(\Delta)=0$. From $\operatorname{Re}(\Delta)=0$, we obtain

$$
4\left(\gamma^{2}-m^{2}-h_{x}^{2}\right)^{3}=27 \gamma^{2} h_{x}^{4},
$$



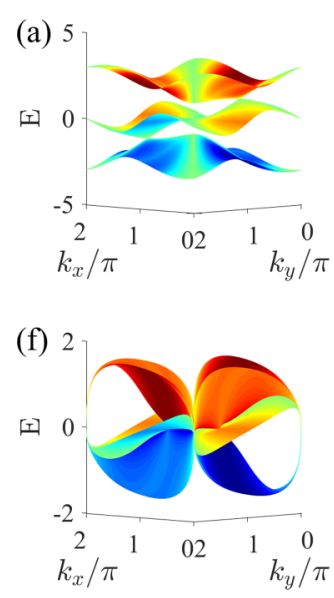
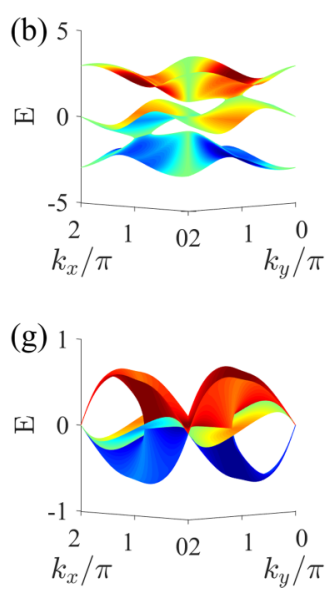
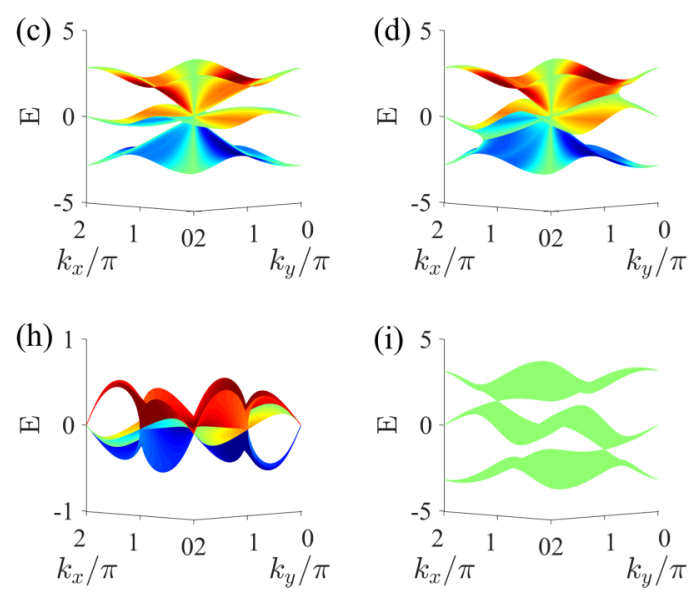

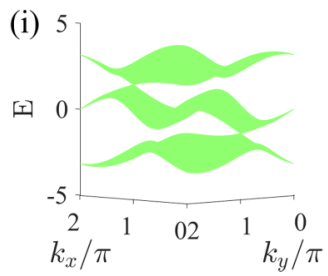

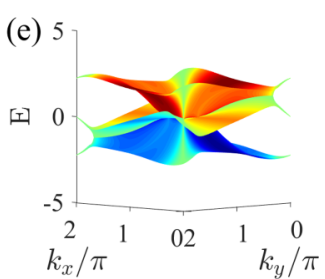

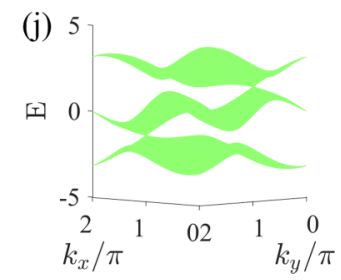

FIG. 9. Energy band structures of the non-Hermitian Hamiltonian in the momentum space as the counterpart of Fig. 8. In all panels, the vertical axis represents the real part of eigenvalues and the color indicates the imaginary part of eigenvalues. (a) $m=1, \gamma=0.388$; (b) $m=1, \gamma=0.5$; (c) $m=0.5, \gamma=0.5$; (d) $m=1, \gamma=1$; (e) $m=1, \gamma=2$; (f) $m=1, \gamma=3$; (g) $m=1, \gamma=4$; (h) $m=1, \gamma=4.162$; (i) $m=-\sqrt{2}, \gamma=0$; and (j) $m=\sqrt{2}, \gamma=0$. The other system parameter is $\kappa=1$.

which determines the green and yellow regions of the phase diagram in Fig. 2. The boundary $\left(\gamma^{2}-m^{2}-4\right)^{3}=108 \gamma^{2}$ in green and the boundary $|\gamma|=|m|$ enclose these two regions with four EP2s. At these four EP2s, the coalesced band energies are imaginary with $\operatorname{Re}(E)=0$. The representative configurations are shown in Figs. 8(d)-(h), showing the movement and merging of EPs. Four EP2s move along the two dashed lines $k_{x}=\pi$ and $k_{y}=\pi$ in Figs. 8(e)-(g) until they merge into two EP2s at $\left(k_{x}, k_{y}\right)=(0, \pi),(\pi, 0)$ at the green boundary in Fig. 8(h) or merge as one EP at $\left(k_{x}, k_{y}\right)=(\pi, \pi)$ at $|\gamma|=|m|$ in Fig. 8(d). The energy bands for Fig. 8 are shown in Fig. 9, where the bulk Fermi arc is observed.

In the phase diagram, the cyan region has four EP2s at $k_{x}=k_{y}$; the green region has two EP2s at $k_{x}=k_{y}$, two EP3s at $k_{x}=-k_{y}$, and four EP2s at $k_{x}=\pi$ and $k_{y}=\pi$; the yellow region has four EP2s at $k_{x}=\pi$ and $k_{y}=\pi$. Under the OBC, the edge states exist in the gapless region in cyan; they are the remnant of topological features inherited from the Hermitian Lieb lattice; however, the edge state does not exist in the gapless regions in green and yellow. At even larger gain and loss, the non-Hermitian Lieb lattice enters the trivial phase.

\section{CONCLUSION}

To conclude, we have proposed an anisotropic 2D nonHermitian Lieb lattice, which has gain and loss along the horizontal and vertical directions, respectively. The nonreciprocal intracell coupling creates nontrivial topology. The gain and loss result in active and dissipative topological edge states with net gain and net loss, respectively. The non-Hermitian Lieb lattice also supports passive topological edge states. Interestingly, the gain and loss can alter the localization position of the corner state. When the magnetic flux enclosed in the unit cell is $\pi / 2$, the corner states are always in one corner of the lattice; however, when the magnetic flux enclosed in the unit cell is $-\pi / 2$, the corner states slowly evolve into the opposite corner as the non-Hermiticity increases. The interplay between the magnetic flux and non-Hermiticity produces rich band structures featured from different types of EPs. The topological properties and rich band structures of the nonHermitian Lieb lattice benefit our understanding of three-band lattice and robust non-Hermitian transport.

\section{ACKNOWLEDGMENTS}

We acknowledge the support of the National Natural Science Foundation of China (Grants No. 11975128, No. 11975166, and No. 11874225). X.Z.Z. is also supported by the Program for Innovative Research at the University of Tianjin (Grant No. TD13-5077).
[1] S. K. Gupta, Y. Zou, X.-Y. Zhu, M.-H. Lu, L.-J. Zhang, X.-P. Liu, and Y.-F. Chen, Adv. Mater. 32, 1903639 (2020).

[2] W. Hu, S. Kaiser, D. Nicoletti, C. R. Hunt, I. Gierz, M. C. Hoffmann, M. Le Tacon, T. Loew, B. Keimer, and A. Cavalleri, Nat. Mater. 13, 705 (2014).

[3] S. Kaiser, C. R. Hunt, D. Nicoletti, W. Hu, I. Gierz, H. Y. Liu, M. Le Tacon, T. Loew, D. Haug, B. Keimer et al., Phys. Rev. B 89, 184516 (2014).

[4] M. Mitrano, A. Cantaluppi, D. Nicoletti, S. Kaiser, A. Perucchi, S. Lupi, P. Di Pietro, D. Pontiroli, M. Riccó, S. R. Clark et al., Nature (London) 530, 461 (2016).
[5] A. Cantaluppi, M. Buzzi, G. Jotzu, D. Nicoletti, M. Mitrano, D. Pontiroli, M. Riccó, A. Perucchi, P. Di Pietro, and A. Cavalleri, Nat. Phys. 14, 837 (2018).

[6] S. A. Sato, P. Tang, M. A. Sentef, U. D. Giovannini, H. Hübener, and A. Rubio, New J. Phys. 21, 093005 (2019).

[7] J. W. McIver, B. Schulte, F.-U. Stein, T. Matsuyama, G. Jotzu, G. Meier, and A. Cavalleri, Nat. Phys. 16, 38 (2020).

[8] C. Booker, B. Buča, and D. Jaksch, arXiv:2005.05062.

[9] J. Tindall, F. Schlawin, M. Buzzi, D. Nicoletti, J. R. Coulthard, H. Gao, A. Cavalleri, M. A. Sentef, and D. Jaksch, Phys. Rev. Lett. 125, 137001 (2020). 
[10] X. Z. Zhang, L. Jin, and Z. Song, Phys. Rev. A 95, 052122 (2017).

[11] H. Shen and L. Fu, Phys. Rev. Lett. 121, 026403 (2018).

[12] T. Yoshida, R. Peters, and N. Kawakami, Phys. Rev. B 98, 035141 (2018).

[13] X. Z. Zhang, L. Jin, and Z. Song, Phys. Rev. B 101, 224301 (2020).

[14] X. Z. Zhang and Z. Song, Phys. Rev. B 102, 174303 (2020).

[15] M. Nakagawa, N. Tsuji, N. Kawakami, and M. Ueda, Phys. Rev. Lett. 124, 147203 (2020).

[16] M. Nakagawa, N. Kawakami, and M. Ueda, Phys. Rev. Lett. 126, 110404 (2021).

[17] S. Longhi, Phys. Rev. B 100, 125157 (2019).

[18] K. Kawabata, K. Shiozaki, M. Ueda, and M. Sato, Phys. Rev. X 9, 041015 (2019).

[19] X. Z. Zhang and Z. Song, Phys. Rev. A 99, 012113 (2019).

[20] X.-W. Luo and C. Zhang, Phys. Rev. Lett. 123, 073601 (2019).

[21] C. Yuce and H. Ramezani, Phys. Rev. A 100, 032102 (2019).

[22] L. Du, J.-H. Wu, M. Artoni, and G. C. La Rocca, Phys. Rev. A 100, 012112 (2019).

[23] Z.-Y. Ge, Y.-R. Zhang, T. Liu, S.-W. Li, H. Fan, and F. Nori, Phys. Rev. B 100, 054105 (2019).

[24] S. Lieu, Phys. Rev. B 100, 085110 (2019).

[25] H. Jiang, R. Lü, and S. Chen, Eur. Phys. J. B 93, 125 (2020).

[26] Z. Xu, R. Zhang, S. Chen, L. Fu, and Y. Zhang, Phys. Rev. A 101, 013635 (2020).

[27] Y.-J. Wu, C.-C. Liu, and J. Hou, Phys. Rev. A 101, 043833 (2020).

[28] C.-H. Liu, K. Zhang, Z. Yang, and S. Chen, Phys. Rev. Research 2, 043167 (2020).

[29] Z. Xu and S. Chen, Phys. Rev. B 102, 035153 (2020).

[30] C. C. Wojcik, X.-Q. Sun, T. Bzdušek, and S. Fan, Phys. Rev. B 101, 205417 (2020).

[31] K. Kawabata, N. Okuma, and M. Sato, Phys. Rev. B 101, 195147 (2020).

[32] W.-X. Cui, L. Qi, Y. Xing, S. Liu, S. Zhang, and H.-F. Wang, Opt. Express 28, 37026 (2020).

[33] S. Li, M. Liu, F. Li, and B. Liu, Phys. Scr. 96, 015402 (2020).

[34] Q.-B. Zeng, Y.-B. Yang, and Y. Xu, Phys. Rev. B 101, 020201(R) (2020).

[35] H. Wu and J.-H. An, Phys. Rev. B 102, 041119(R) (2020).

[36] C.-X. Guo, X.-R. Wang, C. Wang, and S.-P. Kou, Phys. Rev. B 101, 144439 (2020).

[37] D.-W. Zhang, Y.-L. Chen, G.-Q. Zhang, L.-J. Lang, Z. Li, and S.-L. Zhu, Phys. Rev. B 101, 235150 (2020).

[38] D.-W. Zhang, L.-Z. Tang, L.-J. Lang, H. Yan, and S.-L. Zhu, Sci. China Phys. Mech. Astron. 63, 267062 (2020).

[39] X. Zhang and J. Gong, Phys. Rev. B 101, 045415 (2020).

[40] B. R. Zhou, R. Wang, and B. G. Wang, Phys. Rev. B 102, 205116 (2020).

[41] K. Yokomizo and S. Murakami, Phys. Rev. Research 2, 043045 (2020).

[42] L. Wang, Q. Liu, and Y. Zhang, Chin. Phys. B 30, 020506 (2021).

[43] L.-J. Lang, Y. Weng, Y. Zhang, E. Cheng, and Q. Liang, Phys. Rev. B 103, 014302 (2021).

[44] K. Xu, X. Zhang, K. Luo, R. Yu, D. Li, and H. Zhang, Phys. Rev. B 103, 125411 (2021).

[45] J. D. H. Rivero and L. Ge, Phys. Rev. B 103, 014111 (2021).
[46] J. Hou, Y.-J. Wu, and C. Zhang, Phys. Rev. A 103, 033305 (2021).

[47] S. Longhi, Phys. Rev. B 103, 054203 (2021).

[48] S. Schiffer, X.-J. Liu, H. Hu, and J. Wang, Phys. Rev. A 103, L011302 (2021).

[49] X. Zhang, G. Li, Y. Liu, T. Tai, R. Thomale, and C. H. Lee, Commun. Phys. 4, 47 (2021).

[50] S. Yao and Z. Wang, Phys. Rev. Lett. 121, 086803 (2018).

[51] K. Zhang, Z. Yang, and C. Fang, Phys. Rev. Lett. 125, 126402 (2020).

[52] Z. Yang, K. Zhang, C. Fang, and J. Hu, Phys. Rev. Lett. 125, 226402 (2020).

[53] K.-I. Imura and Y. Takane, Phys. Rev. B 100, 165430 (2019).

[54] F. Song, S. Yao, and Z. Wang, Phys. Rev. Lett. 123, 246801 (2019).

[55] F. Song, S. Yao, and Z. Wang, Phys. Rev. Lett. 123, 170401 (2019).

[56] K. Yokomizo and S. Murakami, Phys. Rev. Lett. 123, 066404 (2019).

[57] H. Jiang, L.-J. Lang, C. Yang, S.-L. Zhu, and S. Chen, Phys. Rev. B 100, 054301 (2019).

[58] Y. Yi and Z. Yang, Phys. Rev. Lett. 125, 186802 (2020).

[59] S. Longhi, Phys. Rev. Research 1, 023013 (2019).

[60] H. Wang, J. Ruan, and H. Zhang, Phys. Rev. B 99, 075130 (2019).

[61] D. S. Borgnia, A. J. Kruchkov, and R.-J. Slager, Phys. Rev. Lett. 124, 056802 (2020).

[62] S. Longhi, Phys. Rev. Lett. 124, 066602 (2020).

[63] N. Okuma, K. Kawabata, K. Shiozaki, and M. Sato, Phys. Rev. Lett. 124, 086801 (2020).

[64] B. Zhu, Y. Ke, H. Zhong, and C. Lee, Phys. Rev. Research 2, 023043 (2020).

[65] Q.-B. Zeng and Y. Xu, Phys. Rev. Research 2, 033052 (2020).

[66] K. Kawabata, M. Sato, and K. Shiozaki, Phys. Rev. B 102, 205118 (2020).

[67] N. Silberstein, J. Behrends, M. Goldstein, and R. Ilan, Phys. Rev. B 102, 245147 (2020).

[68] X.-R. Wang, C.-X. Guo, and S.-P. Kou, Phys. Rev. B 101, 121116(R) (2020).

[69] R. Koch and J. C. Budich, Eur. Phys. J. D 74, 70 (2020).

[70] S. R. Pocock, P. A. Huidobro, and V. Giannini, Nanophotonics 8, 1337 (2019).

[71] M. Ezawa, Phys. Rev. B 99, 121411(R) (2019).

[72] X. Zhu, H. Wang, S. K. Gupta, H. Zhang, B. Xie, M. Lu, and Y. Chen, Phys. Rev. Research 2, 013280 (2020).

[73] L. Li, C. H. Lee, and J. Gong, Phys. Rev. Lett. 124, 250402 (2020).

[74] X. Yin and C. Peng, Photon. Res. 8, B25 (2020).

[75] C. Qin, B. Wang, Z. J. Wong, S. Longhi, and P. Lu, Phys. Rev. B 101, 064303 (2020).

[76] K. Sone, Y. Ashida, and T. Sagawa, Nat. Commun. 11, 5745 (2020).

[77] L. Xiao, T. Deng, K. Wang, G. Zhu, Z. Wang, W. Yi, and P. Xue, Nat. Phys. 16, 761 (2020).

[78] Y. Ota, K. Takata, T. Ozawa, A. Amo, Z. Jia, B. Kante, M. Notomi, Y. Arakawa, and S. Iwamoto, Nanophotonics 9, 547 (2020).

[79] B.-Y. Sun and Z.-W. Zhou, Phys. Rev. A 102, 023501 (2020).

[80] B. Zhu, H. Zhong, J. Jia, F. Ye, and L. Fu, Phys. Rev. A 102, 053510 (2020). 
[81] X.-X. Zhang and M. Franz, Phys. Rev. Lett. 124, 046401 (2020).

[82] B. Wang, T. Chen, and X. Zhang, Laser Photonics Rev. 14, 2000092 (2020).

[83] M. Parto, Y. G. N. Liu, B. Bahari, M. Khajavikhan, and D. N. Christodoulides, Nanophotonics 10, 403 (2021).

[84] M. V. Berry, Czechoslovak J. Phys. 54, 1039 (2004).

[85] W. D. Heiss, J. Phys. A: Math. Theor. 45, 444016 (2012).

[86] T. E. Lee, Phys. Rev. Lett. 116, 133903 (2016).

[87] M.-A. Miri and A. Alù, Science 363, eaar7709 (2019).

[88] Y.-X. Xiao, K. Ding, R.-Y. Zhang, Z. H. Hang, and C. T. Chan, Phys. Rev. B 102, 245144 (2020).

[89] N. Goldman, D. F. Urban, and D. Bercioux, Phys. Rev. A 83, 063601 (2011).

[90] D. Guzmán-Silva, C. Mejía-Cortés, M. A. Bandres, M. C. Rechtsman, S. Weimann, S. Nolte, M. Segev, A. Szameit, and R. A. Vicencio, New J. Phys. 16, 063061 (2014).

[91] R. A. Vicencio, C. Cantillano, L. Morales-Inostroza, B. Real, C. Mejía-Cortés, S. Weimann, A. Szameit, and M. I. Molina, Phys. Rev. Lett. 114, 245503 (2015).

[92] S. Taie, H. Ozawa, T. Ichinose, T. Nishio, S. Nakajima, and Y. Takahashi, Sci. Adv. 1, e1500854 (2015).

[93] S. Mukherjee, A. Spracklen, D. Choudhury, N. Goldman, P. Öhberg, E. Andersson, and R. R. Thomson, Phys. Rev. Lett. 114, 245504 (2015).

[94] C. E. Whittaker, E. Cancellieri, P. M. Walker, D. R. Gulevich, H. Schomerus, D. Vaitiekus, B. Royall, D. M. Whittaker, E. Clarke, I. V. Iorsh et al., Phys. Rev. Lett. 120, 097401 (2018).

[95] J. Vidal, R. Mosseri, and B. Douçot, Phys. Rev. Lett. 81, 5888 (1998).

[96] C. Wu, D. Bergman, L. Balents, and S. Das Sarma, Phys. Rev. Lett. 99, 070401 (2007).

[97] J. T. Chalker, T. S. Pickles, and P. Shukla, Phys. Rev. B 82, 104209 (2010).
[98] V. Apaja, M. Hyrkäs, and M. Manninen, Phys. Rev. A 82, 041402(R) (2010).

[99] A. Bermudez, T. Schaetz, and D. Porras, Phys. Rev. Lett. 107, 150501 (2011).

[100] J. D. Bodyfelt, D. Leykam, C. Danieli, X. Yu, and S. Flach, Phys. Rev. Lett. 113, 236403 (2014).

[101] A. Julku, S. Peotta, T. I. Vanhala, D.-H. Kim, and P. Törmä, Phys. Rev. Lett. 117, 045303 (2016).

[102] P. Shukla, Phys. Rev. B 98, 054206 (2018).

[103] M. Goda, S. Nishino, and H. Matsuda, Phys. Rev. Lett. 96, 126401 (2006).

[104] M. Maksymenko, A. Honecker, R. Moessner, J. Richter, and O. Derzhko, Phys. Rev. Lett. 109, 096404 (2012).

[105] S. Peotta and P. Törmä, Nat. Commun. 6, 8944 (2015).

[106] V. J. Kauppila, F. Aikebaier, and T. T. Heikkilä, Phys. Rev. B 93, 214505 (2016).

[107] H. Shen, B. Zhen, and L. Fu, Phys. Rev. Lett. 120, 146402 (2018).

[108] B. Peng, Ş. K. Özdemir, F. Lei, F. Monifi, M. Gianfreda, G. L. Long, S. Fan, F. Nori, C. M. Bender, and L. Yang, Nat. Phys. 10, 394 (2014).

[109] I. Rotter and J. P. Bird, Rep. Prog. Phys. 78, 114001 (2015).

[110] H. Zhou, C. Peng, Y. Yoon, C. W. Hsu, K. A. Nelson, L. Fu, J. D. Joannopoulos, M. Soljačić, and B. Zhen, Science 359, 1009 (2018).

[111] X. Z. Zhang, L. Jin, and Z. Song, Phys. Rev. A 87, 042118 (2013).

[112] L. Jin, P. Wang, and Z. Song, Sci. Rep. 6, 32919 (2016).

[113] T. Yoshida, R. Peters, N. Kawakami, and Y. Hatsugai, Phys. Rev. B 99, 121101(R) (2019).

[114] P. Delplace, T. Yoshida, and Y. Hatsugai, arXiv:2103.08232.

[115] T. Bessho, K. Kawabata, and M. Sato, JPS Conf. Proc. 30, 011098 (2020).

[116] K. Kawabata, T. Bessho, and M. Sato, Phys. Rev. Lett. 123, 066405 (2019). 